\title{
The Increased Transforming Growth Factor- $\beta$ Signaling Induced by Diabetes Protects Retinal Vessels
}

\author{
Zeina Dagher, ${ }^{* \dagger}$ Chiara Gerhardinger, ${ }^{* \dagger}$ Joseph Vaz, ${ }^{\star}$ Michael Goodridge, ${ }^{*}$ Francesco Tecilazich, ${ }^{* \dagger}$ and Mara Lorenzi ${ }^{* \dagger}$
}

From the Schepens Eye Research Institute, ${ }^{*}$ Massachusetts Eye and Ear, Boston; and the Department of Ophthalmology, ${ }^{\dagger}$ Harvard Medical School, Boston, Massachusetts

\author{
Accepted for publication \\ November 15, 2016. \\ Address correspondence to \\ Mara Lorenzi, M.D., Schepens \\ Eye Research Institute, \\ Massachusetts Eye and Ear, \\ 20 Staniford St., Boston, \\ MA 02114. E-mail: mara \\ lorenzi@meei.harvard.edu.
}

\begin{abstract}
The roles of transforming growth factor (TGF)- $\beta$ in extracellular matrix production and vascular remodeling, coupled with increased TGF- $\beta$ expression and signaling in diabetes, suggest TGF- $\beta$ as an important contributor to the microangiopathy of diabetic retinopathy and nephropathy. To investigate whether increased TGF- $\beta$ signaling could be a therapeutic target for preventing retinopathy, we used a pharmacologic approach (SM16, a selective inhibitor of the type 1 TGF- $\beta$ receptor activin receptor-like kinase 5 , orally active) to inhibit the increased, but not the basal, Tgf- $\beta$ signaling in retinal vessels of diabetic rats. At the level of vascular gene expression, 3.5 months' diabetes induced minimal changes. Diabetes + SM16 for 3 weeks caused widespread changes in gene expression poised to enhance vascular inflammation, thrombosis, leakage, and wall instability; these changes were not observed in control rats given SM16. The synergy of diabetes and SM16 in altering gene expression was not observed in the lung. At the level of vascular network morphology, 7 months' diabetes induced no detectable changes. Diabetes + SM16 for 3 weeks caused instead distorted morphology and decreased density. Thus, in diabetes, retinal vessels become dependent on a small increase in TGF- $\beta$ signaling via activin receptor-like kinase 5 to maintain early integrity. The increased TGF- $\beta$ signaling may protect against rapid retinopathy progression and should not be a target of inhibitory interventions. (Am J Pathol 2017, 187: 627-638; http://dx.doi.org/10.1016/j.ajpath.2016.11.007)
\end{abstract}

Retinopathy is a common and dreaded complication of diabetes that can cause sight impairment and blindness. The incidence and rate of progression of diabetic retinopathy have been reduced substantially in the past 2 decades by the implementation of intensive treatment of hyperglycemia, targeting blood glucose levels as near-normal as possible. However, the means of controlling hyperglycemia are still imperfect. In addition, retinal vessels are exquisitely sensitive to even mild elevations in blood glucose levels, as documented in populations studies as by a steep increase in the prevalence of diabetic retinopathy at $\mathrm{HbA}_{1 \mathrm{c}}$ values of $6.5 \%$, just slightly above normal. ${ }^{1}$ This combination of facts explains why retinopathy still develops, even in patients who have received for decades intensive diabetes care. ${ }^{2}$ Hence the ongoing search for adjunct strategies that can prevent or limit the pathogenic effects of residual hyperglycemia on retinal vessels.
In the retina as in other organs, diabetes causes a microangiopathy characterized by a thickening of vascular basement membranes and accelerated apoptosis of pericytes and endothelial cells, leading to vascular wall remodeling and the appearance of microaneurysms, leakage, and eventually acellular capillaries that are nonperfused and herald retinal ischemia. $^{3}$ The roles of transforming growth factor (TGF)- $\beta$ in vascular homeostasis and pathologies have proposed this cytokine as a contributor to, if not a master-mediator of, the microvascular abnormalities caused by diabetes. TGF- $\beta$ regulates the production of extracellular matrix, the replication and survival of endothelial cells, the interactions of

Supported by NIH grant EY017637 (C.G. and M.L.), NIH National Eye Institute Core grant P30EY003790, and the George and Frances Levin Endowment (M.L.).

Disclosures: None declared. 
endothelial cells and pericytes to ensure vessel stability, and the remodeling of vessels. ${ }^{4,5}$ When TGF- $\beta$ signaling to vascular cells is increased-as in Marfan syndrome and other inherited TGF- $\beta$ vasculopathies - vessels show excess extracellular matrix, tortuosity, and aneurysms. ${ }^{6}$ In experimentally diabetic animals, Tgf- $\beta$ synthesis is increased in the kidney, and treatment with anti-Tgf- $\beta$ antibodies prevents the early manifestations of nephropathy. ${ }^{7}$ Tgf- $\beta$ expression and signaling are also increased in the retinal vessels of diabetic rats, and drugs that prevent retinopathy in diabetic rats via different mechanisms of action were concordant in preventing increased Tgf- $\beta$ signaling in the retinal vessels. ${ }^{8}$

Thus, diabetic retinal microangiopathy could be, at least in part, a consequence of excess TGF- $\beta$. We wished to test this possibility using an approach with translational potential, which implied choosing a pharmacologic, as opposed to a genetic, intervention so as to block only the increased Tgf- $\beta$ signaling induced by diabetes, without affecting constitutive signaling required for the homeostasis of adult retinal vessels. ${ }^{9}$ TGF- $\beta$ signals via the combination of types I and II serine/threonine kinase receptors ${ }^{5}$ that form a heteromeric complex on ligand binding. The classic and broadly expressed type I receptor is activin receptor-like kinase (ALK)-5, and uses Smad2 and -3 as intracellular effectors; the other type I receptor is ALK1, which is restricted to the endothelium and activates Smad1, -5 , and -8 . We targeted Alk5 using SM16, a small-molecule kinase inhibitor that occupies the ATP binding site of Alk5, and potently inhibits its receptor function and the phosphorylation of Smad2 and $-3 .{ }^{10}$ SM16 has provided insights on the role of excess TGF- $\beta$ signaling in models of cancer, ${ }^{11}$ vascular fibrosis, ${ }^{10}$ lung fibrosis, ${ }^{12}$ and heart failure ${ }^{13}$ and could be appropriately used as a selective inhibitor of the increased Alk5 signaling in the retinal vessels of diabetic rats on several accounts: i) the ALK5 receptor is present in both retinal pericytes and endothelial cells, ${ }^{14}$ and its abundance in rat retinal vessels is increased by diabetes ${ }^{8}$; ii) retinal endothelial cells also express ALK1, ${ }^{14}$ but SM16 does not inhibit ALK1 signaling ${ }^{10}$; iii) even though SM16 does inhibit ALK4, a type I receptor used by activin, the expression of the activin ligands for Alk4 was undetectable in genome-wide gene profiling of rat retinal vessels ${ }^{8}$ (the array used in that study did not report a sequence for Alk4, but to our knowledge there is no published literature on ALK4 signaling in the adult retina); and iv) with regard to non-ALK kinases, the weak inhibitory activity of SM16 against p38/stress-activated protein kinase $2 \mathrm{a}$ and Raf kinases measured in vitro ${ }^{10}$ was not detected in animal experiments using relatively large doses of SM16. ${ }^{13}$ In addition to its satisfactory characteristics as an inhibitor, SM16 is orally active and has shown an excellent safety profile up to 26 weeks of administration. ${ }^{12}$

Insofar as diabetes increases TGF- $\beta$ and TGF- $\beta$ signaling in the retinal vessels but not in the neural retina, ${ }^{8}$ and vascular cells contribute to retinal cellularity only in small proportion, we performed our studies on isolated retinal vascular preparations.

\section{Materials and Methods}

\section{Animals}

The procedures involving animals conformed to guidelines and were approved by the institutional animal use and care committee. Male Sprague-Dawley rats (5 weeks of age; Taconic Farms, Hudson, NY) were randomly assigned to a diabetic or control group. Diabetes was induced with i.v. streptozotocin $(55 \mathrm{mg} / \mathrm{kg}$ body weight), and insulin was administered to prevent catabolism while maintaining hyperglycemia, as previously described. ${ }^{8}$ SM16 (a gift from Biogen Idec, Cambridge, MA) was given in the chow for 3 weeks to rats with 3 or 6.5 months' duration of diabetes; the 3-month experiments also included control animals treated with SM16. The concentration of SM16 in the chow was adjusted to account for daily food consumption, which was double in the diabetic as compared to control rats, and to yield in all groups a dose of $6 \mathrm{mg} / \mathrm{kg}$ body weight per day. ${ }^{12}$ The rats were euthanized after approximately 3.5 or 7 months of diabetes, along with age-matched controls. Blood, obtained by cardiac puncture, was used for measuring glucose (One Touch UltraMini; LifeScan Inc., Wayne, PA) and $\mathrm{HbA}_{1 \mathrm{c}}$ (Glyc-Affin GHb assay; PerkinElmer, Akron, $\mathrm{OH})$. Eyes were immediately enucleated and processed for the isolation of the whole retina or retinal vessels.

\section{Isolation of the Vascular Network from Fresh Retinas}

Fresh retinal microvessels were isolated by hypotonic lysis of the neural and glial retina, as previously described. ${ }^{8}$ Briefly, retinas were immediately isolated from enucleated eyes and placed in ice-cold autoclaved water for 1 hour. Debris and DNA from the hypotonically lysed neural and glial cells were then washed away with a 5-minute $2 \%$ DNase I (type 2; Sigma-Aldrich, St. Louis, MO) incubation followed by ice-cold phosphate-buffered saline washes for 30 minutes, with gentle pipetting. The intact fresh microvessels were processed for either protein or RNA immediately after isolation. The purity of the preparations was measured (see Western Blot Analysis) on the basis of the enrichment in caveolin-1, previously shown in rat retinal microvessels to be equivalent to the enrichment in structural endothelial proteins such as occludin. ${ }^{15}$ At variance with other endothelial markers, caveolin-1 is not abundantly present in blood elements that, by remaining randomly trapped in vessels, are potential confounders in the quantitative assessments of vascular proteins.

\section{Isolation of the Vascular Network from Fixed Retinas (Retinal Trypsin Digests)}

Retinal trypsin digests were prepared as previously described. ${ }^{15,16}$ Eyes were fixed in $10 \%$ formalin for 3 to 7 days. The retinas were isolated, washed in glycine buffer overnight to remove formalin, and digested in a $0.1 \mathrm{~mol} / \mathrm{L}$ 
Tris buffer solution, $\mathrm{pH} 7.8$, containing $3 \%$ trypsin and $0.2 \mathrm{~mol} / \mathrm{L} \mathrm{NaF}$, for 1 hour. The microvascular networks were cleared from neural and glial cell debris under a dissecting microscope, mounted on slides treated with triethoxysilane (Sigma-Aldrich), and allowed to dry before storage at $-20^{\circ} \mathrm{C}$.

\section{Transferase-Mediated dUTP Nick-End Labeling Reaction}

Retinal trypsin digests were studied with the In Situ Cell Death Detection Kit, Fluorescein (Roche, Mannheim, Germany) to investigate the occurrence of pericyte and endothelial cell apoptosis. As in prior work, ${ }^{15-17}$ nuclei positive for transferase-mediated dUTP nick-end labeling were counted by masked observers and attributed to pericytes or endothelial cells on the basis of shape and topography. Counts of cells positive for transferase-mediated dUTP nick-end labeling are presented per whole retina.

\section{Immunohistochemistry Analysis}

Retinal cryosections were used for confirming caveolin-1 localization selectively at blood vessels. The cryosections $(6 \mu \mathrm{m})$ were fixed in $10 \%$ formalin for 15 minutes, rehydrated, and blocked in 10\% bovine serum albumin for 30 minutes at room temperature. Sections were then incubated overnight at $4{ }^{\circ} \mathrm{C}$ with caveolin-1 antibodies (N-20, 1:1000; Santa Cruz Biotechnology, Santa Cruz, CA), followed by an anti-rabbit IgG conjugated to Alexa Fluor 488 (Molecular Probes, Eugene, OR). The negative controls were obtained by substituting the primary antibody with an equivalent concentration of normal rabbit IgG.

\section{Western Blot Analysis}

Freshly isolated microvessels and whole retinas were homogenized with radioimmunoprecipitation assay buffer containing protease and phosphatase inhibitors. Protein levels were determined with the bicinchoninic acid method using the Micro BCA Protein Assay Kit (Pierce/ThermoFisher, Waltham, MA); reduced proteins were separated by SDS-PAGE.

To measure the purity of the microvessel preparations, membranes were probed with antibodies to caveolin- 1 (N-20, 1:1000; Santa Cruz) as vascular marker, and $\beta$-actin (monoclonal antibody AC-15, 1:20,000; Sigma-Aldrich) as control for loading; the densitometric values were used for calculating the caveolin- $1 / \beta$-actin ratios. The vascular enrichment of the microvessel preparations was calculated by comparing the values of the caveolin- $1 / \beta$-actin ratio in the microvessels to those in whole retinas. Only preparations with a vascular enrichment of fivefold or greater were included in the studies.

To measure Tgf- $\beta$ signaling, membranes were probed with antibodies to phosphorylated-Smad2 (catalog number 3101, 1:1000; Cell Signaling, Danvers, MA) and, after stripping, antibodies to total Smad2 (catalog number 07-408, 1:1000; Millipore, Billerica, MA). Densitometric values were used for calculating the phosphorylated-Smad2/Smad2 ratio for each individual sample.

To determine the effect of SM16 on p38, phospho-p38 and total p38 were also measured in Western blot analysis similarly to Smad2 (rabbit monoclonal antibody 12F8 and rabbit polyclonal catalog number 9212, both 1:1000; Cell Signaling).

To test whether diabetes or SM16 treatment increased apoptosis, membranes were reacted with caspase- 3 antibody (rabbit monoclonal antibody 8G10, 1:1000; Cell Signaling), and inspected for the presence of the 17-kDa band, indicative of caspase- 3 cleavage and activation.

\section{PCR Array and Real-Time PCR}

Total RNA was extracted from retinal microvessels and lung tissue using the Qiagen Mini RNeasy kit (Qiagen, Hilden, Germany); genomic DNA contamination was eliminated via the on-column DNA digestion, as recommended by the manufacturer. The RNA was reverse-transcribed into cDNA, as previously described. ${ }^{18}$ Gene-expression profiling was performed using the $\mathrm{RT}^{2}$ Profiler Endothelial Cell Biology PCR Array (PARN-015; SABiosciences/Qiagen). The array plates included a panel of housekeeping genes used for normalizing the PCR array data, and the appropriate controls for genomic DNA contamination, RNA quality, and PCR performance. Real-time quantitative qPCR (qPCR) was used for verifying gene-expression changes insofar as many of the gene products of interest were secreted proteins. The limited amount of tissue available from the isolated retinal microvessels precluded the possibility of measuring protein levels. Primers and probes for Tgfb1, Ccl2, Vegfa, End2, Fgf2, Pgf, Id1, and Actb were purchased from Applied Biosystems (Foster City, CA). Relative expressions of genes (as compared to that in the control group) were determined by the comparative $\Delta \Delta C_{\mathrm{T}}$ method using $A c t b$ as the endogenous control. ${ }^{8}$

\section{Fibrin Staining, Acellular Capillaries, and Capillary Density}

The retinal vascular preparations in the trypsin digests were stained using the Lendrum Picro-Mallory method to detect thrombi. This method stains fibrin clear red, red blood cells orange, collagen blue, nuclei dark blue/black, and muscle a pale red. Positive controls (not shown) were obtained by staining human thrombus on slides kindly gifted by Dr. J.R. Stone, Department of Pathology, Massachusetts General Hospital (Boston, MA). Acellular capillaries were identified and counted on the basis of specific characteristics of length and diameter (absence of cell nuclei along segments that were at least $40 \mu \mathrm{m}$ in length and of a width equal to at least $20 \%$ of an average capillary). ${ }^{15}$ Capillary density was measured at $20 \times$ magnification in three fields in midretina, using the AxioVision software package version 4 
(Carl Zeiss, Göttingen, Germany). Within each field (275 × $367 \mu \mathrm{m}=$ approximately $0.1 \mathrm{~mm}^{2}$ ), the measuring tool was guided over the length of each individual capillary, yielding a total length per field. Large vessels were not included. The counts from the three fields were averaged for each retinal trypsin digest and expressed as microns of capillary length per $0.1 \mathrm{~mm}^{2}$.

\section{Statistical Analysis}

Data from Western blots and qPCR are presented as means \pm SD. Results among groups were compared using analysis of variance, followed by the Fisher protected least significant difference test (StatView software version 5.0; SAS Institute, Cary, NC). A $P$ value $\leq 0.05$ was considered as evidence of a statistically significant difference between groups. The PCR array gene-expression data were analyzed using the online Excel-based PCR array data analysis templates (SABiosciences). Levels of gene expression were considered significantly different between two groups if the change in the expression level was $\geq 2$-fold and $P$ was $<0.05$; synergy was defined as a statistically significant effect of the combination of diabetes and SM16 treatment in the absence of a significant effect of either perturbation applied singly. To test for synergy between the effects of diabetes and of SM16 in decreasing retinal capillary density, we used a linear regression model with capillary density as the dependent variable and diabetes, SM16, and diabetes-SM16 cross-product as independent variables.

\section{Results}

\section{Dose, Timing, and Selectivity of SM16 To Prevent Increased TGF- $\beta$ Signaling in Retinal Vessels}

The retinal vascular preparations tested throughout the experiments manifested on average an 11-fold enrichment in the endothelial marker caveolin-1 when compared to the neural retina; such satisfactory enrichment was comparable in all experimental groups (Figure 1).

To identify a dose of SM16 sufficient for inhibiting in retinal vessels increased Tgf- $\beta$ signaling without affecting constitutive signaling, we first tested the effects of drug against those of exogenous TGF- $\beta$. TGF- $\beta$ injected systemically increased Smad2 phosphorylation in rat retinal vessels, and the increase was prevented (Figure 2) by oral SM16 administered at a concentration of $0.15 \mathrm{~g} / \mathrm{kg}$ of chow, yielding in normal rats a dose of $6 \mathrm{mg} / \mathrm{kg}$ of body weight per day. This dose had been found efficacious against radiationinduced lung injury and fibrosis in rodents, and safe for up to 26 weeks of administration ${ }^{12}$; we therefore used this dose in all subsequent studies with SM16. To time SM16 administration, we examined Tgf- $\beta$ signaling at short intervals after the induction of diabetes to intervene promptly and prevent excess Tgf- $\beta$ activity on the retinal vessels. Increased Smad2 phosphorylation appeared briskly between
2.5 and 3 months after the induction of diabetes (Figure 3, A and B). The administration of SM16 was thus initiated after 3 months of diabetes and continued for 3 weeks to counteract the increase in Tgf- $\beta$ signaling induced by diabetes without affecting basal levels (Figure 3C). Lack of SM16 effect on basal Tgf- $\beta$ signaling was confirmed also in the retinal vessels of control rats (Figure 3D).

To test the selectivity of SM16 for ALK5, we measured the effects of diabetes and SM16 on p38/stress-activated protein kinase 2 . At variance with the increased activity reported in the whole retina of diabetic rats, ${ }^{19}$ in the isolated retinal vessels, p38/Sapk2a activity was not increased by diabetes, and was not affected by SM16 (Figure 4A). Finally, it was important to ascertain whether the effects of SM16 could be attributed to inhibition of Alk5 signaling, as opposed to altered Alk1 signaling consequent to diabetes and Alk5 inhibition. The expression of Idl mRNA, a specific downstream target of Alk 1,5 showed no changes due to diabetes or SM16 treatment (Figure 4B).

These data indicate that the chosen dose and timing of administration of SM16 targeted selectively and promptly the increased Tgf- $\beta$ signaling in diabetic retinal vessels, and that this increased signaling could be attributed to Alk5.

\section{Diabetes and SM16 Synergistically Alter Gene Expression in Retinal Vessels}

To capture the earliest effects of diabetes on the retinal vessels and the impact of TGF- $\beta$ inhibition, we profiled gene expression in rats with 3.5 months of diabetes, a duration insufficient to cause histopathology ${ }^{16}$ or even to accelerate apoptosis ${ }^{18}$ in rat retinal vessels. A group of diabetic rats and a group of control rats received SM16 for 3 weeks; the drug did not affect body weight, blood glucose, or the levels of $\mathrm{HbA}_{1 \mathrm{c}}$ (Table 1). Diabetes of 3.5 months' duration changed significantly the expression of 3 of the 84 genes in the array-Pgf, Fgf2, and Edn2-which were all up-regulated (Table 2 and Figure 5). This finding likely represents a stereotypical response to stress, insofar as the expression of $P g f$ mRNA is increased in many organs and tissues in pathologic conditions ${ }^{20}$ and in the retina in diabetes, ${ }^{21}$ and the up-regulation of $F g f 2$ and $E d n 2$ transcripts accompanies retinal injury or disease. ${ }^{22}$

The administration of SM16 to the diabetic rats had two effects (Table 2). One was the normalization of the levels of the $P g f$ transcript increased by diabetes, consistent with the facts that $P g f$ is a specific transcriptional target of TGF- $\beta$ via $\mathrm{ALK}^{23}$ and that $P g f$ expression can be stimulated by TGF- $\beta{ }^{20}$ The results were confirmed by qPCR analysis (Figure 5). The second effect was the induction of extensive changes in gene expression in the retinal vessels, well beyond those induced by diabetes. On the one hand, SM16 caused a marked up-regulation of the transcript that codes for $\mathrm{C}-\mathrm{C}$ motif chemokine ligand $(\mathrm{Ccl})-2$, a protein that attracts white blood cells and sustains inflammation, and of the transcript that codes for Fas, the regulator of programed 
A

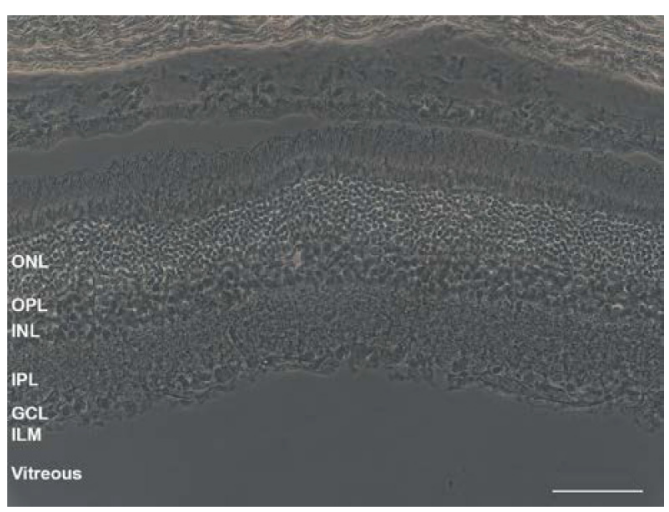

B

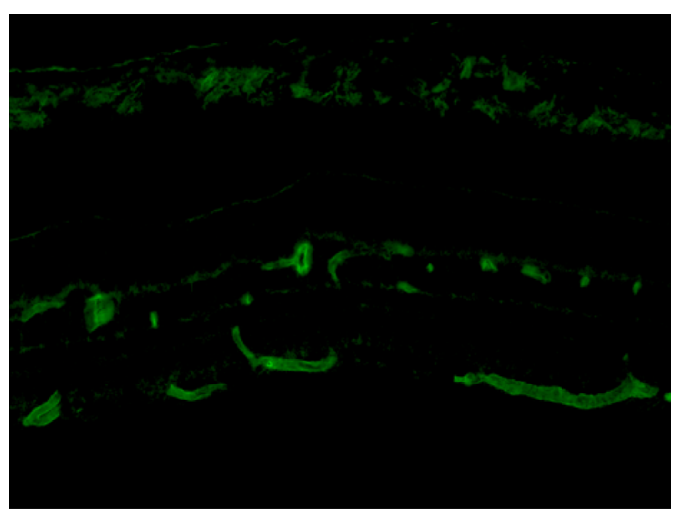

E

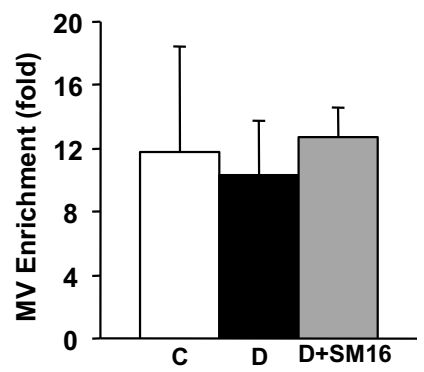

Figure 1 Endothelial enrichment of the retinal vessel preparations as compared to the whole retina. A: Phase contrast image of a rat posterior eye cryosection, showing the retinal layers. B: Caveolin-1 immunohistochemistry analysis in a consecutive cryosection, showing exclusive localization to the retinal vessels, located in the inner two thirds of the retina. C: Western blot measuring caveolin-1 and $\beta$-actin in retinal microvessels and whole retina isolated from control rats $(C)$, rats with a 3.5-month duration of diabetes untreated (D), and diabetic rats treated with SM16 for 3 weeks (D+SM16). D: Bar plot of the caveolin/ $\beta$-actin ratio in the microvessels (MV) and whole retina (WR), showing the greater abundance of caveolin- 1 in the vessels as compared to the whole retina. E: Bar plot showing that the vascular enrichment of the retinal microvessel preparations used for experiments was on average 11 -fold greater than in the whole retina and quantitatively similar among the groups. The enrichment was calculated from the caveolin/ $\beta$-actin ratio in the microvessels divided by the caveolin/ $\beta$-actin ratio in the whole retina for each rat. Data are expressed as means \pm SD in the indicated number of rats. $n=7$ (D, WR); $n=10$ $(\mathbf{E}, \mathrm{D}+\mathrm{SM} 16) ; n=12(\mathbf{E}, \mathbf{C}) ; n=13(\mathbf{E}, \mathrm{D}) ; n=35(\mathbf{D}, \mathrm{MV}) .{ }^{* *} P<0.0001$. Scale bar $=100 \mu \mathrm{m}(\mathbf{A}$ and $\mathbf{B})$. GCL, ganglion cell layer; ILM, inner limiting membrane; INL, inner nuclear layer; IPL, inner plexiform layer; $0 \mathrm{NL}$, outer nuclear layer; $\mathrm{OPL}$, outer plexiform layer.

cell death. On the other hand, SM16 caused extensive down-regulation of transcripts coding for proteins that confer protective functional characteristics to the endothelium (barrier, anticoagulant, vasoactive), provide trophic signals (vascular endothelial growth factor $\mathrm{A}$ and its receptor FMS-related tyrosine kinase 1), and mediate the relationship of the endothelial cells with pericytes (TEK receptor tyrosine kinase) and the extracellular matrix (integrins $\alpha_{5}$ and $\alpha_{\mathrm{V}}$ ). With regard to the extracellular matrix, SM16 also decreased by 3.4-fold the level of the transcript for collagen-18 (Coll8 $\alpha_{1}$ chain; $\left.P=0.05\right)$, a heparan sulfate proteoglycan that is needed for the structural stability of basement membranes ${ }^{24}$ and that was not altered by diabetes.

The effects of SM16 on gene expression by diabetic retinal vessels could not be attributed to generic toxicity of the drug. In control rats, a SM16 dose equivalent to that administered to the diabetic rats and given similarly for 3 weeks did not decrease $\operatorname{Smad} 2 /-3$ phosphorylation (at basal levels in control rats) (Figure 3D), and changed the expression of only 3 of the 84 genes in the profiler array. SM16 increased twofold the level of the Ccl2 mRNA (Table 2), but the change was not confirmed by qPCR; and SM16 decreased twofold the expression of the ILIl7 and Angpt1 mRNAs (data not shown). The Angptl transcript was decreased by SM16 also in the diabetic retinal vessels, albeit not significantly $(-1.61$-fold; $P=0.11)$. The effects of SM16 on endothelial transcripts could not be attributed to widespread endothelial demise either, as was suggested by the selectivity of endothelial-specific transcripts downregulated by SM16 (eg, nitric oxide synthase-3 and E-selectin were not affected), and documented by unchanged indices of apoptosis (Figure 6). Finally, the possibility that the endothelial gene-expression changes induced by SM16 in diabetic rats could reflect changes in Alk1 signaling was not supported by the measurements of IdI mRNA (Figure 4B). Hence, in diabetes, the inhibition of increased ALK5 signaling to retinal vascular cells changes 


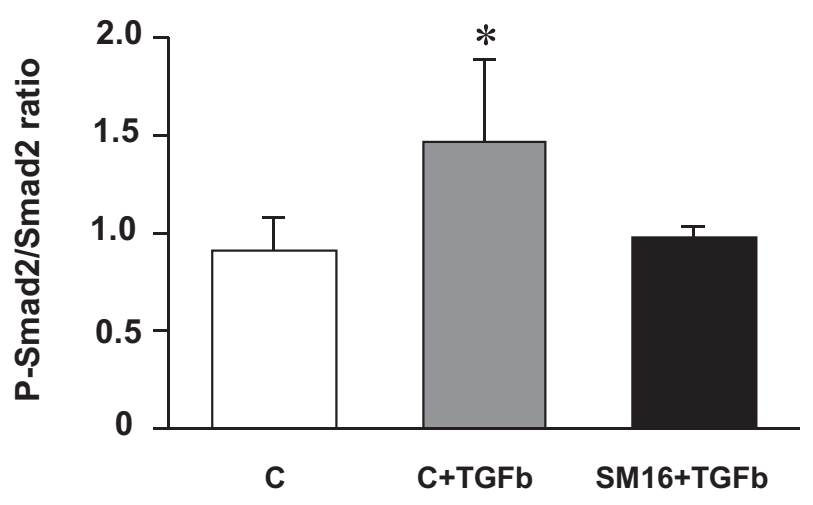

Figure 2 Dose of SM16 to prevent increased, but not basal, transforming growth factor (Tgf)- $\beta$ signaling in retinal vessels. Bar plot of phosphorylated (P)-Smad2/total Smad2 ratio in retinal microvessels in control rats $(C)$, rats injected i.p. with human recombinant active TGF- $\beta_{1}$ (60 $\mu \mathrm{g} ; \mathrm{R} \& \mathrm{D}$ Systems, Minneapolis, MN) and euthanized 1 hour later $(\mathrm{C}+\mathrm{TGFb})$; and rats treated for 5 days with SM16 at $6 \mathrm{mg} / \mathrm{kg}$ per day and then injected with TGF- $\beta_{1}$ (SM16+TGFb). Blots were probed for P-Smad2, stripped, and reprobed for total Smad2 for calculation of the ratio in each individual sample. Data are expressed as means \pm SD. $n=2(\mathrm{SM} 16+\mathrm{TGFb})$; $n=3(\mathrm{C}) ; n=5$ (C+TGFb). ${ }^{*} P<0.05$ versus control.

their biosynthetic profile in the direction of loss of specializations important for vessel stability.

\section{The Effect of Diabetes and SM16 on Vascular Gene Expression Is Site-Specific}

To evaluate the site-specificity of the biosynthetic changes caused by SM16, we tested informative transcripts in the lung of diabetic rats. The lung parenchyma is mostly made of vessels, and endothelial cells are the most represented cell population in the lung parenchyma ( $43 \%$ in the rat). ${ }^{25}$ In the lung, diabetes induced a small increase in $T g f b$ mRNA expression (2.0 relative expression vs 1.0 in control rats; $P=0.03$; data not shown), but the response of other transcripts was discordant from that observed in the retinal microvessels. The $E d n 2$ transcript, measured as an index of stress, was barely detectable in the lung of control rats and undetectable in the lung of diabetic rats, treated or untreated with SM16. This pattern was at sharp variance with the fivefold up-regulation of the $E d n 2$ transcript that was induced by diabetes in the retinal vessels, and further increased by SM16 treatment (Table 2 and Figure 5). The $C c l 2$ transcript, measured as an index of proinflammatory changes, was significantly up-regulated by diabetes in the lung, and SM16 prevented the increase (Figure 7A) - again at sharp variance with the events in retinal vessels, where diabetes alone had not increased $C c l 2$ mRNA expression, but where the combination of diabetes and SM16 treatment had stimulated a fivefold up-regulation (Table 2 and Figure 7B). Thus, the lung tissue and the retinal vessels differed in their response to diabetes as well as in their response to the removal of the diabetesinduced increase in Alk5 signaling.

\section{Diabetes and SM16 Synergistically Alter Morphology and Density of the Retinal Capillary Network}

The changes in endothelial gene expression caused by SM16 in diabetic retinal vessels could activate events such as leukostasis, inflammation, and clotting. To enhance the likelihood of identifying such events and their possible consequences on capillary demise, we studied SM16 in rats with diabetes of 7-month duration, a time at which our model

\section{A}

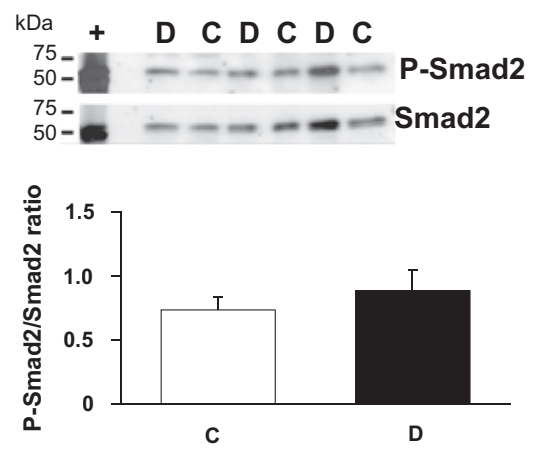

C

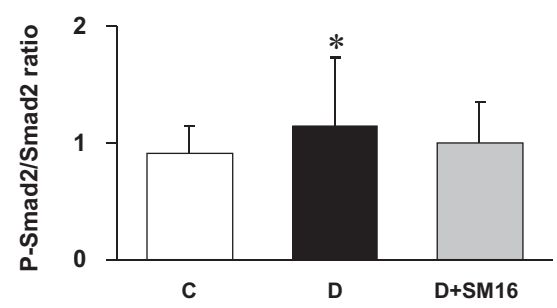

B

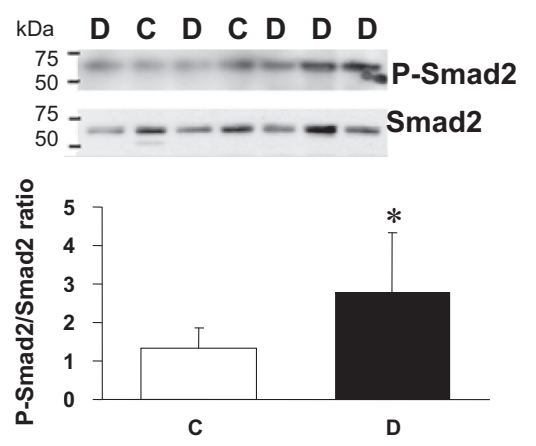

D

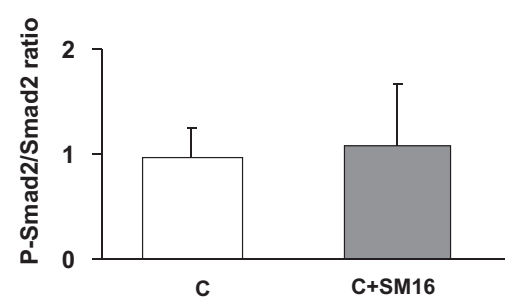

Figure 3 Timing of SM16 administration to prevent increased transforming growth factor (Tgf) $\beta$ signaling induced by diabetes in retinal vessels. A and B: Western blots and bar plots showing that the phosphorylated (P)-Smad2/total Smad2 ratio was not yet increased in diabetic (D) versus control (C) rats after 2.5 months of diabetes (A), but was significantly increased after 3 months of diabetes (B). C and D: SM16 given for 3 weeks to rats with 3 months of diabetes (D+SM16) prevented the increase in P-Smad2/total Smad2 ratio, as shown by the comparison in diabetic versus control rats $(\mathbf{C})$, without affecting the basal ratio in control rats (D). Data are expressed as means \pm SD. $n=4$ $(\mathbf{A}$ and $\mathbf{B}, \mathbf{C}) ; n=5(\mathbf{A}, \mathrm{D}) ; n=7(\mathbf{B}, \mathrm{D}) ; n=9(\mathbf{D}, \mathbf{C}$ and $\mathrm{C}+$ SM16); $n=10$ (C, D+SM16); $n=19$ $(\mathrm{C}, \mathrm{D}) ; n=26(\mathrm{C}, \mathrm{C}) .{ }^{*} P<0.05$ versus control. 
A

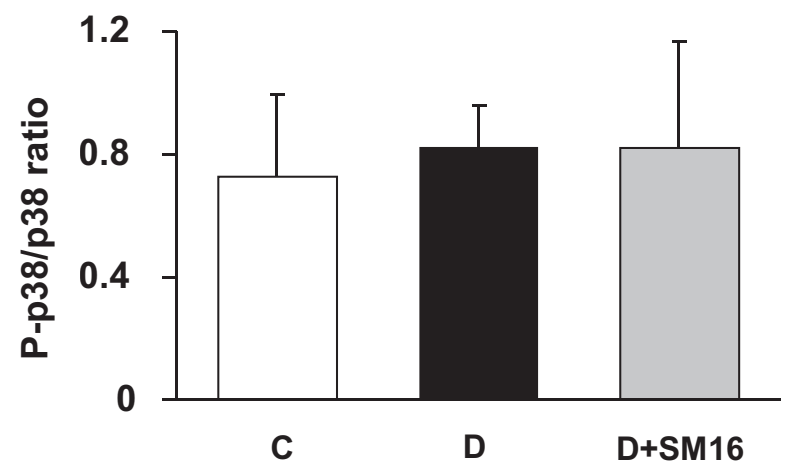

B

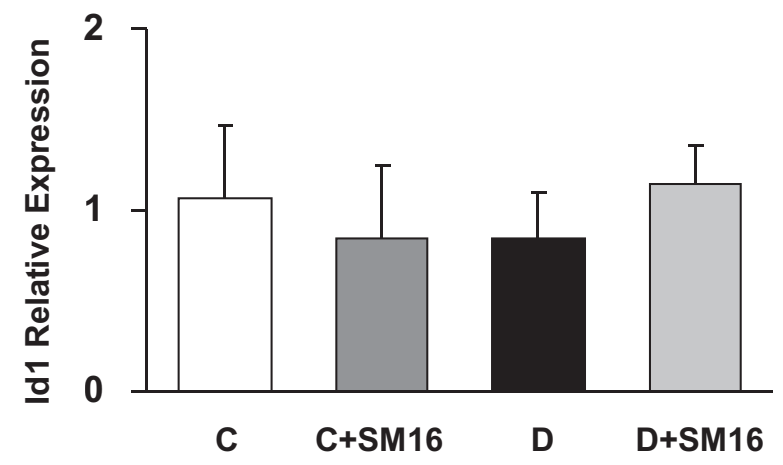

Figure $4 \mathrm{p} 38$ /stress-activated protein kinase (Sapk)-2a and activin receptor-like kinase (Alk1) signaling in diabetic retinal vessels. A: Bar plot of the phosphorylated (P)-p38/total p38 ratio, as measured by Western blot, showing that $\mathrm{p} 38$ activity was not increased by diabetes (D) or altered by SM16 administration (D+SM16), when compared to controls (C). B: Bar plot of the relative expression of inhibitor of DNA-binding 1, HLH protein (Id)-1 measured by real-time quantitative PCR, showing no effect of diabetes or SM16 treatment on Alk1 signaling. Data are expressed as means \pm SD. $n=3$ (A, C and D); $n=4($ A, D+SM16); $n=5$ (B, D+SM16); $n=7$ (B, C+SM16 and D); $n=11(\mathbf{B}, \mathrm{C})$.

of insulin-treated diabetes manifests in retinal vessels extensive biosynthetic changes ${ }^{8}$ but not yet structural abnormalities. $^{15-17}$ In rats with 7 months of diabetes (Table 3), the retinal vessels showed normal morphology and no increase in fibrin thrombi (Figure 8A), and the number of acellular capillaries - an established cumulative index of capillary nonperfusion in the retina ${ }^{16}$-was $1.5 / \mathrm{mm}^{2}$ of retina (median, 0 to 2 range), not yet different from the number in control rats treated or untreated with SM16
Table 2 Effects of Diabetes and SM16 on Gene Expression in Rat Retinal Vessels

\begin{tabular}{llll}
\hline & \multicolumn{2}{l}{ Fold regulation vs control } \\
\cline { 2 - 4 } Gene & D & D+SM16 & C+SM16 \\
\hline Pgf (placental growth factor) & $+2.35^{*}$ & +1.29 & +1.01 \\
Fgf2 (fibroblast growth factor 2) & $+4.53^{*}$ & $+3.32^{*}$ & +1.16 \\
Edn2 (endothelin 2) & $+5.48^{*}$ & $+9.19^{*}$ & +1.25 \\
Ccl2 [chemokine (C-C motif) ligand 2] & +1.42 & $+6.08^{*}$ & $+2.75^{*}$ \\
Fas (Fas cell surface death receptor) & +1.93 & $+2.72^{*}$ & -1.51 \\
Cdh5 (cadherin 5) & -1.39 & $-5.02^{*}$ & -1.08 \\
Ocln (occludin) & -1.06 & $-3.46^{*}$ & -1.46 \\
Pecam1 (platelet/endothelial cell & -1.14 & $-4.02^{*}$ & +1.15 \\
$\quad$ adhesion molecule 1) & & & \\
Tek/Tie2 (TEK receptor tyrosine & -1.46 & $-3.46^{*}$ & -1.23 \\
$\quad$ kinase) & & & \\
Vegfa (vascular endothelial growth & -1.33 & $-2.76^{*}$ & -1.38 \\
$\quad$ factor A) & & & \\
Flt1 (FMS-related tyrosine kinase 1) & -1.19 & $-6.86^{*}$ & +1.41 \\
Itga5 (integrin- $\alpha_{5}$ ) & -1.16 & $-7.25^{*}$ & -1.18 \\
Itgav (integrin- $\alpha$ V) & +1.02 & $-2.53^{*}$ & -1.92 \\
End1 (endothelin-1) & -1.05 & $-2.47^{*}$ & +1.04 \\
Endra (endothelin receptor type A) & -1.11 & $-2.01^{*}$ & -1.39 \\
Agt (angiotensinogen) & -1.18 & $-8.01^{*}$ & -1.31 \\
Thbd (thrombomodulin) & -1.22 & $-3.53^{*}$ & -1.49 \\
Tfpi (tissue factor pathway inhibitor) & +1.80 & $-6.70^{*}$ & +1.78 \\
\hline
\end{tabular}

Data are expressed as fold-changes in the regulation of genes in the retinal vessels of diabetic rats $(D ; n=5)$, diabetic rats treated with SM16 $(D+S M 16 ; n=6)$, and control rats treated with SM16 $(C+S M 16 ; n=6)$, as compared to control rats $(C ; n=9)$, among the 84 genes represented in the endothelial PCR array.

*Statistically significant change, defined as meeting the combined criteria of a twofold or greater change in expression level, with a significance level of $P<0.05$.

$\left(0.25 / \mathrm{mm}^{2}\right.$ of retina, 0 to 2 range $)$. In the diabetic rats treated with SM16, the identification of thrombi and of acellular capillaries was precluded by the profoundly disturbed morphology of the retinal vessels (Figure 8A). The capillaries were stretched to become of uniformly thin caliber, obscuring the visibility of the luminal content, and the network appeared less dense. The capillary density in the retinal trypsin digests of the diabetic rats treated with SM16 was significantly lower than in untreated diabetic rats (total capillary length, $4653 \pm 62$ versus $5788 \pm 371 \mu \mathrm{m} / 0.1 \mathrm{~mm}^{2}$; $P=0.04)$ and tended to be lower than in control rats $(5187 \pm 594)$ and in control rats treated with SM16 (5615 \pm 688 ) (both, $P=0.10$ ) (Figure 8B). The coefficient

Table 1 Body Weight, Blood Glucose, and Glycosylated Hemoglobin Levels in Rats after 3.5 Months of Diabetes

\begin{tabular}{lllr}
\hline Group & Weight, $g$ & Blood glucose, mg/dL & Glycosylated hemoglobin, \% \\
\hline C & $534 \pm 61(n=66)$ & $201 \pm 87(n=43)$ & $5.8 \pm 1.1(n=63)$ \\
D & $352 \pm 85(n=41)^{* * *}$ & $543 \pm 87(n=29)^{* * *}$ & $11.6 \pm 1.8(n=39)^{* * *}$ \\
D+SM16 & $342 \pm 68(n=26)^{* * *}$ & $571 \pm 45(n=18)^{* * *}$ & $12.4 \pm 2.4(n=26)^{* * *}$ \\
C+SM16 & $507 \pm 19(n=22)$ & $176 \pm 55(n=20)$ & $6.0 \pm 1.3(n=22)$ \\
\hline
\end{tabular}

Data are expressed as means \pm SD.

$* * * P<0.001$ versus controls and $\mathrm{C}+\mathrm{SM} 16$.

C, control rats; $C+S M 16 D$, control rats treated with SM16; D, diabetic rats; D+SM16, diabetic rats treated with SM16. 
A

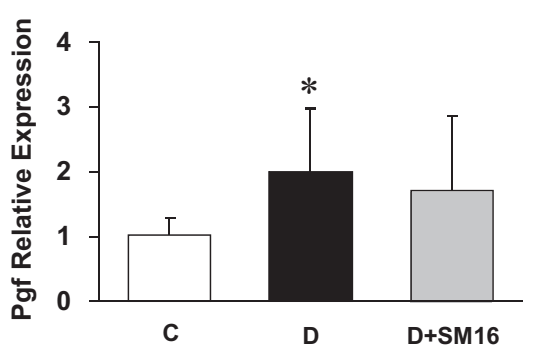

B

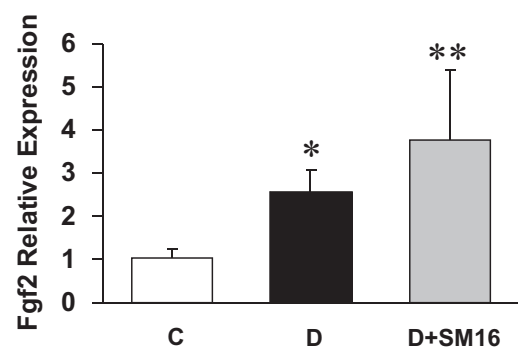

C

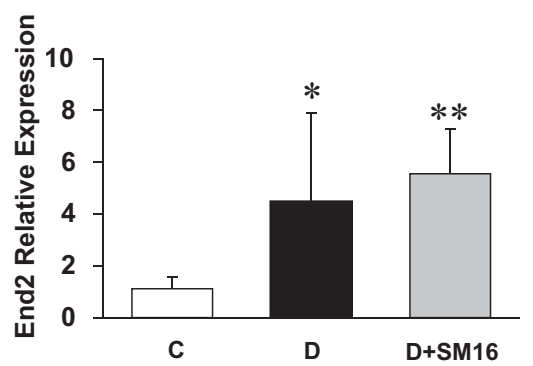

Figure 5 Verification of gene expression changes induced by diabetes and SM16 in retinal vessels. Relative expression of Pgf (A), Fgf2 (B), and End2 (C), as measured by real-time quantitative PCR, in retinal microvessels of control (C) and diabetic (D) rats and diabetic rats treated with SM16 (D+SM16). Data are expressed as means \pm SD. $n=4$ to 7 rats per group. ${ }^{*} P<0.05,{ }^{*} P<0.01$ versus control.

of interaction of diabetes and SM16 in causing decreased capillary density was consistent with a synergistic interaction $(P=0.05)$. Insofar as the control rats treated with SM16 did not show grossly abnormal vascular patterns, the disturbed morphology, decreased mechanical stability, and decreased density of retinal capillaries in the diabetic rats treated with SM16 appear attributable to the effect of SM16 precluding the diabetes-induced increase in ALK5 signaling.

\section{Discussion}

Collectively, our observations indicate that, in diabetes, the retinal vessels become dependent on an increase in TGF- $\beta$ signaling via ALK5 to maintain early integrity. The increased TGF- $\beta$ signaling caused by diabetes is of small magnitude, consistent with the type of changes induced by a chronic and insidious disease, but appears to have an adaptive value, in particular in the stabilization of vessels, one of the crucial functions of TGF- $\beta .^{26}$

We used a pharmacologic inhibitor of Alk5 signaling, at a dose that satisfied the requirements for both specificity and selective inhibition of the increased Tgf- $\beta$ signaling induced by diabetes, without affecting constitutive signaling. In fact, in the retinal vessels of normal rats, the dose of SM16 did not measurably alter Smad2 phosphorylation, did not cause morphologic alterations, and resulted in only three changes in
A

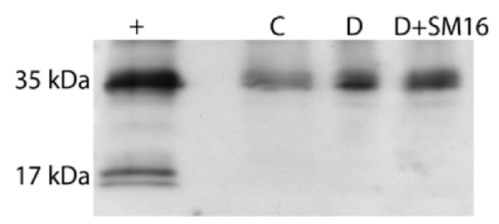

B
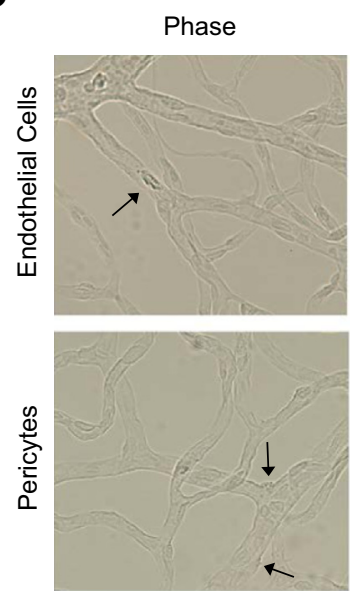

Figure 6 Indices of apoptosis in the retinal vessels of diabetic rats untreated or treated with SM16. Caspase- 3 activation was used to test for massive vascular cell apoptosis after 3.5 months of diabetes, and transferase-mediated dUTP nick-end labeling (TUNEL) positivity to enumerate individual apoptotic cells after 7 months of diabetes. A: Western blot of caspase- 3 in retinal microvessels ( $30 \mu \mathrm{g}$ of lysate/lane from a pool of six microvessel preparations for each group) showing absence of caspase-3 activation in control (C) and diabetic (D) rats, and in diabetic rats treated with SM16 (D+SM16). Active caspase-3 is identified as a $17-\mathrm{kDa}$ band in the positive $(+)$ control lane (camptothecin-treated Jurkat lysate; BD Biosciences, San $\infty$ Jose, CA). B: Images of retinal trypsin digests, showing TUNEL positivity of an elongated endothelial cell .ّ. nucleus (arrow, top row), and of two round pericyte nuclei (arrows, bottom row). C: Bar plot of the number of TUNEL-positive endothelial cells and pericytes counted per retina, showing no effect of diabetes or SM16. Data are expressed as means \pm SD. $n=6$ to 8 rats per group.

C

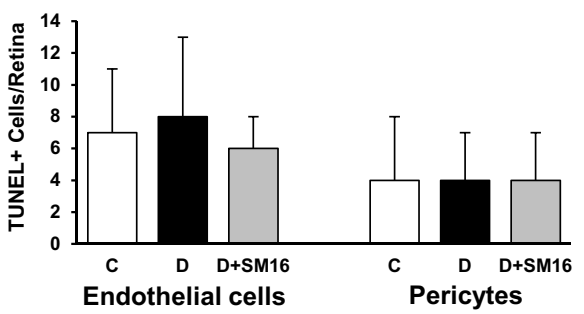


A

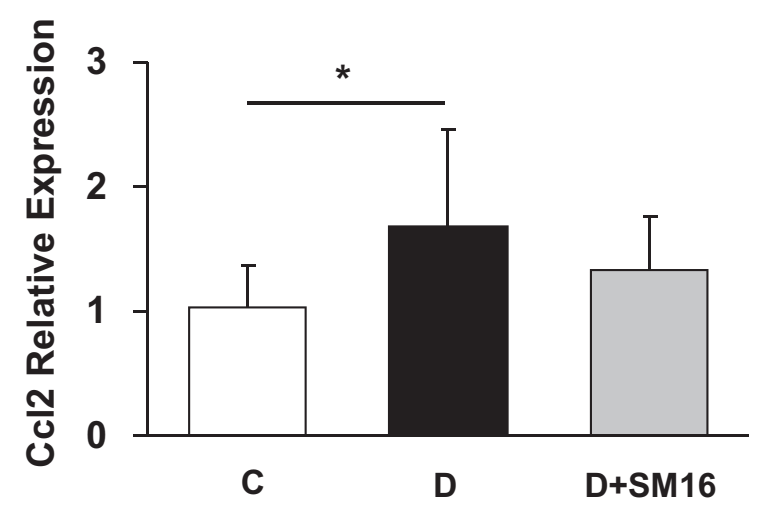

B

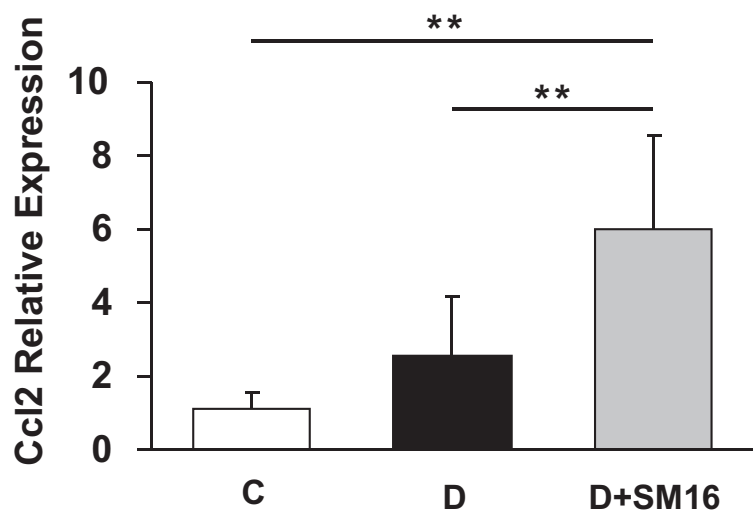

Figure 7 Comparison of the effects of diabetes and SM16 on chemokine ( $\mathrm{C}-\mathrm{C}$ motif) ligand $(\mathrm{Ccl})-2$ expression in retinal vessels and lung tissue. $\mathbf{A}$ and $\mathbf{B}$ : Bar plots of the relative expression of $\mathrm{Ccl} 2$ measured by real-time quantitative PCR showing $(\mathbf{A})$ in lung tissue a pattern of response to diabetes and to SM16 treatment different from (B) the pattern in retinal microvessels. Data are expressed as means \pm SD. $n=5$ to 8 rats per group. ${ }^{*} P<0.05,{ }^{*} P<0.01$. C, control; $\mathrm{D}$, diabetes; $\mathrm{D}+\mathrm{SM} 16$, diabetes + SM16.

gene expression. It is noteworthy that one of the changes was the down-regulation of angiopoietin-1, which is produced mostly, if not only, by pericytes. This finding suggests an exquisite sensitivity of normal retinal pericytes to ALK-5 inhibition, consistent with observations in tumor neovessels. ${ }^{27}$

However, when SM16 was given to diabetic rats and deprived the retinal vessels of the small increment in Tgf- $\beta$ signaling induced by diabetes, the vessels showed an image of profound distress, similar to that observed in normal murine vessels after sustained inhibition of constitutive Tgf- $\beta$ signaling by receptor deletion or receptor antagonists. Whether tested in embryonic, ${ }^{28}$ newborn, ${ }^{29}$ or adult ${ }^{9}$ mice, these interventions have caused vessels to become leaky and unstable, often totally or partially collapsed, and nonperfused. Indeed, the vascular phenotype resulting from conditional deletion of the Tgf- $\beta$ type II receptor in the eyes of newborn mice shows most of the characteristic features of diabetic retinopathy. ${ }^{29}$ The biosynthetic changes that we observed in the retinal vessels of the diabetic rats treated with SM16 provide molecular mechanisms for the above abnormalities. In our study, SM16 treatment undermined in retinal vessels at least three stabilizing influences: i) the angiopoietin $1 /$ TEK receptor tyrosine kinase paracrine loop ${ }^{26}$; ii) integrins $\alpha_{5}$ and $\alpha_{\mathrm{V}}$, the combined absence of which causes extensive defects in vessel stability and remodeling $^{30}$; and iii) collagen-18, which has a structural role in maintaining basement membrane integrity. The combination of these effects likely accounts for the decreased mechanical stability and decreased density of retinal vessels that we observed in the diabetic rats treated with SM16. In addition, the down-regulation by SM16 of anticoagulant molecules and the up-regulation of $\mathrm{Ccl} 2$, also called monocyte chemoattractant protein-1 and a major promoter of inflammation, may have contributed to the capillary demise.

Evidence is mounting that the increased TGF- $\beta$ expression and signaling observed in vascular pathologies are mechanisms of protection that vessels activate against stress. The evidence published to date relates to the aorta. In an angiotensin II-induced mouse model of abdominal aortic aneurysm, the aneurysm-prone segment showed increased Tgf- $\beta$ expression, ${ }^{31}$ and systemic neutralization of the Tgf- $\beta$ activity markedly accelerated the progression of the aneurysm and its complications. ${ }^{32}$ In a mouse model of aortic stenosis and heart failure induced by aortic banding, there was increased Tgf- $\beta$ signaling, and treatment with SM16-despite reducing myocardial fibrosis and ameliorating function-eventually accelerated the death of the mice by causing aortic rupture at the banded site. ${ }^{13}$ The investigators of both studies concluded that intervening against the increased Tgf- $\beta$ signaling attenuated a protective response. Our observations now add diabetic retinal vessels to the settings in which increased TGF- $\beta$ signaling has a protective role required for the preservation of vessel integrity.

Table 3 Body Weight, Blood Glucose, and Glycosylated Hemoglobin Levels in Rats after 7 Months of Diabetes

\begin{tabular}{lllr}
\hline Group & Weight, g & Blood glucose, mg/dL & Glycated hemoglobin, \% \\
\hline C & $623 \pm 43(n=8)$ & $161 \pm 34(n=6)$ & $6.7 \pm 0.5(n=8)$ \\
D & $342 \pm 47(n=7)^{* * *}$ & $551 \pm 51(n=7)^{* * *}$ & $14.0 \pm 1.1(n=7)^{* * *}$ \\
D+SM16 & $341 \pm 49(n=7)^{* * *}$ & $551 \pm 34(n=7)^{* * *}$ & $14.1 \pm 1.1(n=7)^{* * *}$ \\
\hline
\end{tabular}

Data are expressed as means \pm SD.

$* * * P<0.001$ versus controls.

C, control rats; D, diabetic rats; D+SM16, diabetic rats treated with SM16. 
A
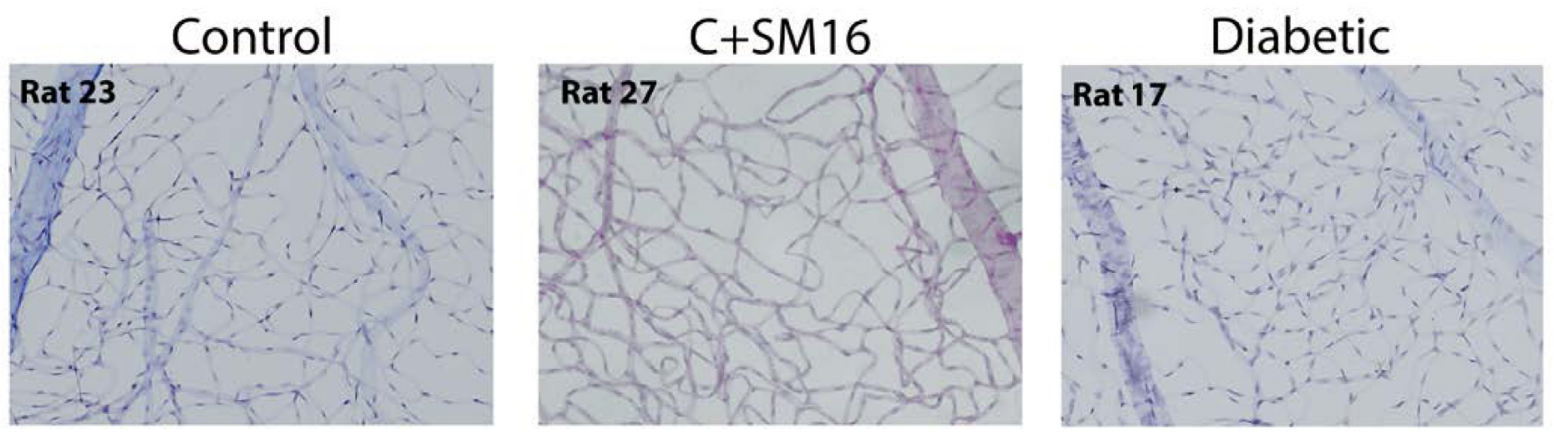

\section{$\mathrm{D}+\mathrm{SM} 16$}
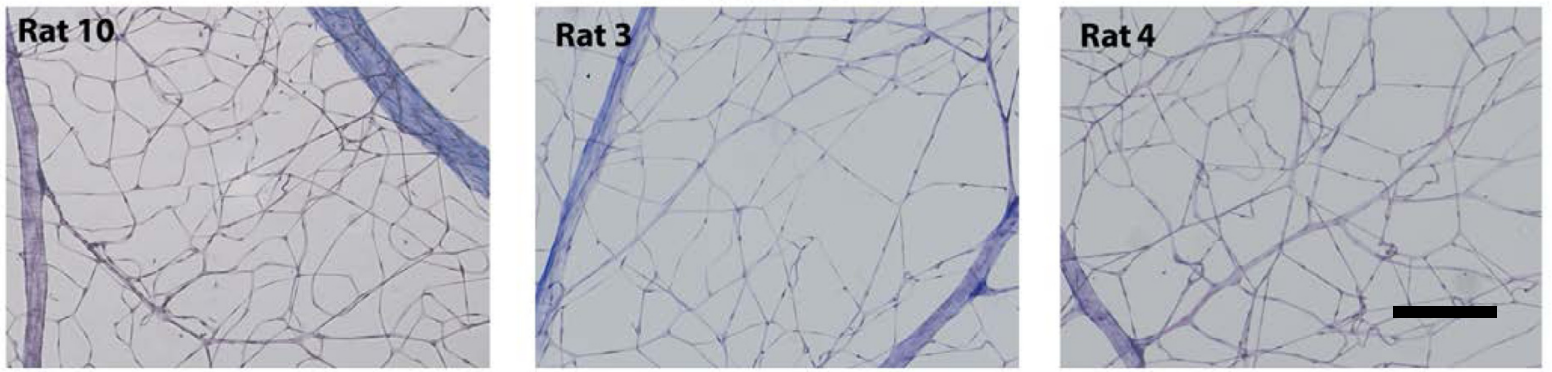

B

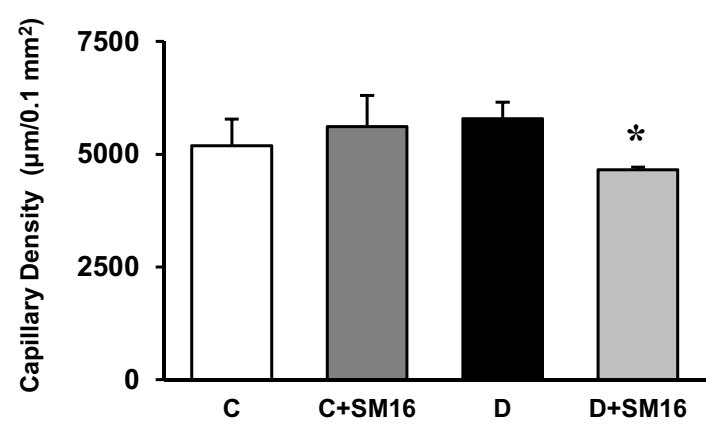

In fact, retinal vessels may be especially dependent on a small increment in TGF- $\beta$ signaling under stressful conditions, also to be protected against inflammation. This concept is suggested by the differential behavior of proinflammatory $\mathrm{Ccl} 2$ that we observed in the retinal vessels as compared to the lung. Although we did not verify that the lung CCL2 response to diabetes reflects TGF- $\beta$ signaling via ALK5, this concept is highly likely. As in diabetes, also in inflammatory lung pathology, the CCL2 overexpression associated with increased TGF- $\beta$ is corrected by ALK5 inhibitors. ${ }^{33}$ A greater sensitivity of retinal than lung vascular cells to the deprivation of ALK5 signaling is consistent with the severe consequences that endothelial deletion of ALK5 has on the vessels of the brain but not the lung. ${ }^{28}$ A reason for this may be that the retina and the brain are immuneprivileged regions, ${ }^{34}$ and as such are less tolerant of
Figure 8 Diabetes and SM16 synergistically alter morphology and density of the retinal capillary network. A: Representative images of retinal trypsin digests, stained using the Lendrum Picro-Mallory method for fibrin, showing normal morphology and density in control rats (C), control rats treated for 3 weeks with SM16 (C+SM16), and diabetic rats with 7 months of diabetes untreated (D), but abnormal vessel morphology, geometry, and density in diabetic rats with 7 months of diabetes treated with SM16 for 3 weeks $\infty$ (D+SM16). All images were taken in midsection of the retinas. B: Bar plot of capillary density (total capillary length per field) in retinal trypsin digests, showing decreased capillary density in D+SM16 rats. Capillary length was measured using the AxioVision software package version 4 (Carl Zeiss) in three areas of midretina, covering $0.1 \mathrm{~mm}^{2}$; in each rat, the lengths measured in the three areas were averaged to yield one value used in the computation. Data are expressed as means \pm SD. $n=4$ to 5 rats per group. ${ }^{*} P<0.05$ versus diabetes. Scale bar $=100 \mu \mathrm{m}$.

interferences with the regulation of TGF- $\beta$, which is a key anti-inflammatory molecule and an important contributor to immune privilege. ${ }^{35}$

Our findings do not exclude that inhibition of increased TGF- $\beta$ signaling in diabetes may lead to some beneficial effects. In the mouse model of diabetic nephropathy, the prevention of the increased Tgf- $\beta$ signaling has been reported to prevent several early renal abnormalities. ${ }^{7}$ In our studies of retinal vessels after short-term diabetes, SM16 treatment prevented the overexpression of $P g f$, an abnormality that when induced in normal eyes causes some vascular and retinal changes similar to those of early diabetic retinopathy. ${ }^{21}$ After a longer diabetes duration, normalized Tgf- $\beta$ signaling may also limit basement membrane thickening. However, the data from the models of aortic aneurysms ${ }^{32}$ and aortic stenosis, ${ }^{13}$ combined with our 
data in diabetic retinal vessels, indicate that interference with the matrix-preserving and vessel-stabilizing actions of TGF- $\beta$ can - at least in some organs-drive the balance of the effects of TGF- $\beta$ inhibition in a direction that is clinically undesirable.

The novel concept of an adaptive TGF- $\beta$ response to diabetes that protects retinal vessels may have clinical relevance. On the one hand, it will need to be taken into account during evaluation of the rationale and modalities for targeting TGF- $\beta$ therapeutically in diabetes. On the other hand, it provides insights usable for better understanding the course of human diabetic retinopathy. An insufficient TGF- $\beta$ response to diabetes may facilitate the progression of retinopathy; conversely, a robust response may decelerate its course and limit its severity. Our observations, together with reports that certain polymorphisms in the TGF- $\beta_{1}$ gene are associated with increased risk and severity of human diabetic retinopathy, ${ }^{36,37}$ suggest a deserving venue for investigations to identify modulators of retinopathy.

\section{Acknowledgments}

We thank Dr. Leona E. Ling (Biogen Idec, Cambridge, MA) for providing SM16, and Dr. James R. Stone (Massachusetts General Hospital, Boston, MA) for providing sections of a human thrombus.

\section{References}

1. Nathan DM, Balkau B, Bonora E, Borch-Johnsen K, Buse JB, Colagiuri S, Davidson MB, DeFronzo R, Genuth S, Holman RR, Ji L, Kirkman S, Knowler WC, Schatz D, Shaw J, Sobngwi E, Steffes M, Vaccaro O, Wareham N, Zinman B, Kahn R; International Expert Committee: International Expert Committee report on the role of the A1C assay in the diagnosis of diabetes. Diabetes Care 2009, 32: $1327-1334$

2. Lachin JM, White NH, Hainsworth DP, Sun W, Cleary PA, Nathan DM; Diabetes Control and Complications Trial (DCCT)/ Epidemiology of Diabetes Interventions and Complications (EDIC) Research Group: Effect of intensive diabetes therapy on the progression of diabetic retinopathy in patients with type 1 diabetes: 18 years of follow-up in the DCCT/EDIC. Diabetes 2015, 64:631-642

3. Archer DB: Bowman Lecture 1998. Diabetic retinopathy: some cellular, molecular and therapeutic considerations. Eye 1999, 13(Pt 4): 497-523

4. Leask A, Abraham DJ: TGF-beta signaling and the fibrotic response. FASEB J 2004, 18:816-827

5. Lebrin F, Deckers M, Bertolino P, Ten Dijke P: TGF-beta receptor function in the endothelium. Cardiovasc Res 2005, 65:599-608

6. Akhurst RJ: The paradoxical TGF-beta vasculopathies. Nat Genet 2012, 44:838-839

7. Ziyadeh FN, Hoffman BB, Han DC, Iglesias-De La Cruz MC, Hong SW, Isono M, Chen S, McGowan TA, Sharma K: Long-term prevention of renal insufficiency, excess matrix gene expression, and glomerular mesangial matrix expansion by treatment with monoclonal antitransforming growth factor-beta antibody in $\mathrm{db} / \mathrm{db}$ diabetic mice. Proc Natl Acad Sci U S A 2000, 97:8015-8020

8. Gerhardinger C, Dagher Z, Sebastiani P, Park YS, Lorenzi M: The transforming growth factor-beta pathway is a common target of drugs that prevent experimental diabetic retinopathy. Diabetes 2009, 58: 1659-1667

9. Walshe TE, Saint-Geniez M, Maharaj AS, Sekiyama E, Maldonado AE, D'Amore PA: TGF-beta is required for vascular barrier function, endothelial survival and homeostasis of the adult microvasculature. PLoS One 2009, 4:e5149

10. Fu K, Corbley MJ, Sun L, Friedman JE, Shan F, Papadatos JL, Costa D, Lutterodt F, Sweigard H, Bowes S, Choi M, BoriackSjodin PA, Arduini RM, Sun D, Newman MN, Zhang X, Mead JN, Chuaqui CE, Cheung HK, Cornebise M, Carter MB, Josiah S, Singh J, Lee WC, Gill A, Ling LE: SM16, an orally active TGF-beta type I receptor inhibitor prevents myofibroblast induction and vascular fibrosis in the rat carotid injury model. Arterioscler Thromb Vasc Biol 2008, 28:665-671

11. Suzuki E, Kim S, Cheung HK, Corbley MJ, Zhang X, Sun L, Shan F, Singh J, Lee WC, Albelda SM, Ling LE: A novel small-molecule inhibitor of transforming growth factor beta type I receptor kinase (SM16) inhibits murine mesothelioma tumor growth in vivo and prevents tumor recurrence after surgical resection. Cancer Res 2007, 67: 2351-2359

12. Anscher MS, Thrasher B, Zgonjanin L, Rabbani ZN, Corbley MJ, Fu K, Sun L, Lee WC, Ling LE, Vujaskovic Z: Small molecular inhibitor of transforming growth factor-beta protects against development of radiation-induced lung injury. Int J Radiat Oncol Biol Phys 2008, 71:829-837

13. Engebretsen KV, Skardal K, Bjornstad S, Marstein HS, Skrbic B, Sjaastad I, Christensen G, Bjornstad JL, Tonnessen T: Attenuated development of cardiac fibrosis in left ventricular pressure overload by SM16, an orally active inhibitor of ALK5. J Mol Cell Cardiol 2014, 76:148-157

14. Van Geest RJ, Klaassen I, Vogels IM, Van Noorden CJ, Schlingemann RO: Differential TGF-\{beta $\}$ signaling in retinal vascular cells: a role in diabetic retinopathy? Invest Ophthalmol Vis Sci 2010, 51:1857-1865

15. Dagher Z, Park YS, Asnaghi V, Hoehn T, Gerhardinger C, Lorenzi M: Studies of rat and human retinas predict a role for the polyol pathway in human diabetic retinopathy. Diabetes 2004, 53:2404-2411

16. Mizutani M, Kern TS, Lorenzi M: Accelerated death of retinal microvascular cells in human and experimental diabetic retinopathy. J Clin Invest 1996, 97:2883-2890

17. Sun W, Gerhardinger C, Dagher Z, Hoehn T, Lorenzi M: Aspirin at low-intermediate concentrations protects retinal vessels in experimental diabetic retinopathy through non-platelet-mediated effects. Diabetes 2005, 54:3418-3426

18. Gerhardinger C, McClure KD, Romeo G, Podesta F, Lorenzi M: IGF-I mRNA and signaling in the diabetic retina. Diabetes 2001, 50:175-183

19. Du Y, Tang J, Li G, Berti-Mattera L, Lee CA, Bartkowski D, Gale D, Monahan J, Niesman MR, Alton G, Kern TS: Effects of p38 MAPK inhibition on early stages of diabetic retinopathy and sensory nerve function. Invest Ophthalmol Vis Sci 2010, 51:2158-2164

20. Fischer C, Mazzone M, Jonckx B, Carmeliet P: FLT1 and its ligands VEGFB and PlGF: drug targets for anti-angiogenic therapy? Nat Rev Cancer 2008, 8:942-956

21. Kowalczuk L, Touchard E, Omri S, Jonet L, Klein C, Valamanes F, Berdugo M, Bigey P, Massin P, Jeanny JC, Behar-Cohen F: Placental growth factor contributes to micro-vascular abnormalization and blood-retinal barrier breakdown in diabetic retinopathy. PLoS One 2011, 6:e17462

22. Rattner A, Nathans J: The genomic response to retinal disease and injury: evidence for endothelin signaling from photoreceptors to glia. J Neurosci 2005, 25:4540-4549

23. Ota T, Fujii M, Sugizaki T, Ishii M, Miyazawa K, Aburatani H, Miyazono K: Targets of transcriptional regulation by two distinct type I receptors for transforming growth factor-beta in human umbilical vein endothelial cells. J Cell Physiol 2002, 193:299-318

24. Seppinen L, Pihlajaniemi T: The multiple functions of collagen XVIII in development and disease. Matrix Biol 2011, 30:83-92 
25. Haies DM, Gil J, Weibel ER: Morphometric study of rat lung cells. I. Numerical and dimensional characteristics of parenchymal cell population. Am Rev Respir Dis 1981, 123:533-541

26. Armulik A, Abramsson A, Betsholtz C: Endothelial/pericyte interactions. Circ Res 2005, 97:512-523

27. Kano MR, Bae Y, Iwata C, Morishita Y, Yashiro M, Oka M, Fujii T, Komuro A, Kiyono K, Kaminishi M, Hirakawa K, Ouchi Y, Nishiyama N, Kataoka K, Miyazono K: Improvement of cancertargeting therapy, using nanocarriers for intractable solid tumors by inhibition of TGF-beta signaling. Proc Natl Acad Sci U S A 2007, 104: 3460-3465

28. Nguyen HL, Lee YJ, Shin J, Lee E, Park SO, McCarty JH, Oh SP: TGF-beta signaling in endothelial cells, but not neuroepithelial cells, is essential for cerebral vascular development. Lab Invest 2011, 91: $1554-1563$

29. Braunger BM, Leimbeck SV, Schlecht A, Volz C, Jagle H, Tamm ER: Deletion of ocular transforming growth factor beta signaling mimics essential characteristics of diabetic retinopathy. Am J Pathol 2015, 185:1749-1768

30. van der Flier A, Badu-Nkansah K, Whittaker CA, Crowley D, Bronson RT, Lacy-Hulbert A, Hynes RO: Endothelial alpha5 and alphav integrins cooperate in remodeling of the vasculature during development. Development 2010, 137:2439-2449

31. Rush C, Nyara M, Moxon JV, Trollope A, Cullen B, Golledge J: Whole genome expression analysis within the angiotensin
II-apolipoprotein E deficient mouse model of abdominal aortic aneurysm. BMC Genomics 2009, 10:298

32. Wang Y, Ait-Oufella H, Herbin O, Bonnin P, Ramkhelawon B, Taleb S, Huang J, Offenstadt G, Combadiere C, Renia L, Johnson JL, Tharaux PL, Tedgui A, Mallat Z: TGF-beta activity protects against inflammatory aortic aneurysm progression and complications in angiotensin II-infused mice. J Clin Invest 2010, 120:422-432

33. Segawa S, Goto D, Yoshiga Y, Sugihara M, Hayashi T, Chino Y, Matsumoto I, Ito S, Sumida T: Inhibition of transforming growth factor-beta signalling attenuates interleukin (IL)-18 plus IL-2-induced interstitial lung disease in mice. Clin Exp Immunol 2010, 160:394-402

34. Niederkorn JY: See no evil, hear no evil, do no evil: the lessons of immune privilege. Nat Immunol 2006, 7:354-359

35. Zhou R, Horai R, Mattapallil MJ, Caspi RR: A new look at immune privilege of the eye: dual role for the vision-related molecule retinoic acid. J Immunol 2011, 187:4170-4177

36. Beranek M, Kankova K, Benes P, Izakovicova-Holla L, Znojil V, Hajek D, Vlkova E, Vacha J: Polymorphism R25P in the gene encoding transforming growth factor-beta (TGF-beta1) is a newly identified risk factor for proliferative diabetic retinopathy. Am J Med Genet 2002, 109:278-283

37. Buraczynska M, Baranowicz-Gaszczyk I, Borowicz E, Ksiazek A: TGF-betal and TSC-22 gene polymorphisms and susceptibility to microvascular complications in type 2 diabetes. Nephron Physiol 2007, 106:p69-p75 